\title{
IDENTIFICATION OF FREEWAY MACROSCOPIC MODELS USING INFORMATION FROM MOBILE PHONES
}

\author{
A. Alessandri, R. Bolla, A.F. Grassia, M. Repetto
}

\begin{abstract}
A method for the identification of a freeway macroscopic model is presented. The model is based on the idea of dividing a freeway trunk in sections covered by the cells of the wireless network and associated with state variables describing density of vehicles, mean velocity, and percentage of active mobile phones. Using the density and hand-off measurements from the cellular network, the parameters of such model are identified via an algorithm based on stochastic approximation. Successful simulation results are reported and discussed.
\end{abstract}

\section{INTRODUCTION}

Different macroscopic models are proposed in the literature to describe the dynamics of the traffic flow (see, for an introduction, [1]). The use of such models is fundamental for the purpose of surveillance and control. A dynamic model allows to represent the evolution of the state variables, which, in general, are the density and the mean speed. Using such model, different control systems were designed that rely on variable speed signalling and ramp metering [2], [3], [4], [5]. More recently, optimal control was proposed to set the speed advise [6]; a recent survey on ramp-metering can be found in [7].

A macroscopic model depend in general on parameters that have to be identified via least-squares techniques. If the model is linear, the identification is quite simple, as one can apply standard least-squares for linear systems. The identification of more complex models, as those we shall consider in the following, require the solution of nonlinear parameter optimization problems that result from the minimization of a least-squares cost function [8], [9]. As to such class of problems, powerful algorithms have been proposed that are based on stochastic approximation with well-established convergence properties and, among them, the simultaneous perturbation stochastic approximation (SPSA) [10], [11], [12]. The essential feature of SPSA is the underlying gradient approximation that requires, at each iteration step, only two measurements of the cost function regardless of the dimension of the optimization problem.

This work was supported by the EU and the Regione Liguria trough the Regional Programmes of Innovative Action (PRAI) of the European Regional Development Fund (ERDF).

A. Alessandri is with the Department of Production Engineering, Thermoenergetics and Mathematical Models, DIPTEMUniversity of Genoa, P.le Kennedy Pad. D, 16129 Genova, Italy alessandriediptem. unige. it

A.F. Grassia is with the Institute of Intelligent Systems for Automation, ISSIA-CNR National Research Council of Italy, Via De Marini 6, 16149 Genova, Italy filippo.grassiaege.issia.cnr.it

R. Bolla and M. Repetto are with the Department of Communications, Computer and System Sciences, DIST-University of Genova, Via Opera Pia 13, 16145 Genova, Italy raffaele.bolla@unige.it repettodist.unige.it
This allows for a significant decrease in the computational effort, especially when a large number of parameters are to be optimized. Moreover, such technique appears essential whenever the gradient of the cost function is difficult to determine.

The measurements of the traffic conditions are usually taken by means of expensive devices such as television cameras and loop detectors (see, for an introduction, [13]). These equipments are located at distance of several kilometers, provide only local information, and may need a lot of lowlevel signal processing. Indeed, the management system of a wireless network that covers a freeway trunk and handles the radio-based communications among the cellular phones aboard moving cars and the base stations can be queried to give global information on the traffic. Unfortunately, such information concerns only cars with an active mobile onboard.

Using a suitable assumption on the uniform distribution of active devices within each section of the freeway trunk, we propose a macroscopic model that describes the dynamics of density, mean speed, and the percentage of cars with an onboard active mobile phone. As to the density, this is a simple balance equation of the flow for each segment. The dynamic of the velocity is derived by the well-known model $D$, which is appropriate to describe the nonlinear phenomena that occur in congested traffic conditions (see, for details, [1]). The dynamic equation for the percentage of vehicles with active mobile phone is formulated via state augmentation. The three state variables are assigned to a section of freeway that is covered by a base station. In practice, the segment between two loop detectors in a freeway model is replaced by a cell of the wireless network. The model parameters are identified by using an SPSA tuning method for its low computational burden and relative ease of implementation [14].

The paper is organized as follows. Section II describes the macroscopic traffic model that enables one to account for the presence of active mobile phones in the cars moving in the freeway. The identification of the model parameters is described in Section III. Simulation setup and results of the numerical tests are reported in Section IV. Conclusions and prospect of future work are discussed in Section V.

\section{A MACROSCOPIC TRAFFIC MODEL BASED ON WIRELESS NETWORK}

A mobile phone communication network is well-suited to providing information on the traffic from the active mobile phones of the drivers. In order to evaluate the kind of 
information that can be collected from the mobiles, a suitable model is required. Toward this end, we can split a freeway trunk in different segments associated with the cells of the wireless network (see Fig. 1).

Referring to the $i$-th segment among $K+1$ ones in Fig. 1 and using a discrete-time representation with sample time equal to $\Delta T$, one can describe the dynamic behavior of the traffic by means of the following state variables

$\rho_{i}(t)$ : density of vehicles at time $t \Delta T$

$v_{i}(t)$ : mean velocity at time $t \Delta T$

$\delta_{i}(t) \in(0,1)$ : percentage of vehicles with active mobiles at time $t \Delta T$,

where $t=0,1, \ldots$ and $i=0,1, \ldots, K$. Moreover, let $d_{i}$ the length of section $i, i=1,2, \ldots, K-1$. In general, there may exist ramps and so we need the input variables

$r_{i}(t)$ : flow of the vehicles entering the $i$-th section by means of on-ramps between time $(t-1) \Delta T$ and time $t \Delta T$

$s_{i}(t)$ : flow of the vehicles coming out of the $i$-th section by means of off-ramps between time $(t-$ 1) $\Delta T$ and time $t \Delta T$

where $t=0,1, \ldots$ and $i=1,2, \ldots, K-1$.

So far, apart from $\delta_{i}(t)$, we rely on the standard set of variables of model $\mathrm{D}$, which is well-suited to describing the nonlinear behavior of freeway traffic [1]. Before going into details, we need to explicitly assume that the distribution of the active mobile phones in each section is uniform and so we have

$$
\begin{aligned}
& \rho_{i}^{c}(t)=\delta_{i}(t) \rho_{i}(t) \\
& q_{i}^{c}(t)=\delta_{i}(t) q_{i}(t)
\end{aligned}
$$

according to the definition of

$q_{i}(t)$ : flow of the vehicles exiting the $i$-th section and entering the $i+1$-th section between time $(t-$ 1) $\Delta T$ and time $t \Delta T$

$\rho_{i}^{c}(t)$ : density of vehicles with active mobile phone in the $i$-th section at time $t \Delta T$

$q_{i}^{c}(t)$ : flow of the vehicles with active mobile phone exiting the $i$-th section and entering the $i+1$-th section between time $(t-1) \Delta T$ and time $t \Delta T$,

where $t=0,1, \ldots$ and $i=0,1, \ldots, K-1$. Moreover, according to model $\mathrm{D}$, the flow among sections is given by

$$
q_{i}(t)=\alpha \rho_{i}(t) v_{i}(t)+(1-\alpha) \rho_{i+1}(t) v_{i+1}(t)
$$

where $t=0,1, \ldots, i=0,1, \ldots, K-1$, and $\alpha \in[0,1]$ is a constant parameter.

The uniformity assumption is supposed to be valid for the ramps as well, and so, after defining

$r_{i}^{c}(t)$ : flow of the vehicles with active mobile phone entering the $i$-th section by means of on-ramps between time $(t-1) \Delta T$ and time $t \Delta T$

$s_{i}^{c}(t)$ : flow of the vehicles with active mobile phone coming out of the $i$-th section by means of offramps between time $(t-1) \Delta T$ and time $t \Delta T$, we assume the following to hold

$$
\begin{aligned}
& r_{i}^{c}(t)=\delta_{i}(t) r_{i}(t), i \in I_{r} \\
& s_{i}^{c}(t)=\delta_{i}(t) s_{i}(t), i \in I_{s}
\end{aligned}
$$

where $t=0,1, \ldots ; I_{r}$ and $I_{s}$ are the index sets of the segments with on-ramps and off-ramps, respectively.

In the following, we implicitly suppose to know the variables $\rho_{i}^{c}(t)$ and $q_{i}^{c}(t)$ (via the hand-off measurements of the cellular network) as well as $r_{i}(t)$ and $s_{i}(t)$ (using either loop detectors or tollgate sensors).

As to the dynamic relationship between the state variables, we first consider the density dynamics [1], which is given by

$$
\rho_{i}(t+1)=\rho_{i}(t)+\frac{\Delta T}{d_{i}}\left[q_{i-1}(t)-q_{i}(t)+r_{i}(t)-s_{i}(t)\right]
$$

where $t=0,1, \ldots$ and $i=1,2, \ldots, K-1$. Using (2), (5) yields

$\rho_{i}(t+1)=\rho_{i}(t)+\frac{\Delta T}{d_{i}}\left[\frac{q_{i-1}^{c}(t)}{\delta_{i-1}(t)}-\frac{q_{i}^{c}(t)}{\delta_{i}(t)}+r_{i}(t)-s_{i}(t)\right]$

where $t=0,1, \ldots, i=1, \ldots K-1$; in addition, $r_{i}(t)=0$ if $i \notin I_{r}$ and $s_{i}(t)=0$ if $i \notin I_{s}$. Moreover, one has to consider the first and last sections, which are usually described by

$$
\begin{aligned}
\rho_{0}(t+1) & =\rho_{0}(t)+\xi_{0}^{\rho}(t) \\
\rho_{K}(t+1) & =\rho_{K}(t)+\xi_{K}^{\rho}(t)
\end{aligned}
$$

where $\xi_{0}^{\rho}(t)$ and $\xi_{K}^{\rho}(t)$ are scalar noises. Secondly, we shall consider the dynamics of the mean speed is that of model D, i.e.,

$$
\begin{aligned}
v_{i}(t+1) & =v_{i}(t)+\frac{\Delta T}{\tau}\left\{V\left[\rho_{i}(t)\right]-v_{i}(t)\right\} \\
& +\frac{\Delta T \zeta}{d_{i}} v_{i}(t)\left[v_{i-1}(t)-v_{i}(t)\right] \\
& -\frac{\nu \Delta T\left[\rho_{i+1}(t)-\rho_{i}(t)\right]}{\tau d_{i}\left[\rho_{i}(t)+\chi\right]}+\xi_{i}^{v}(t)
\end{aligned}
$$

where $\xi_{i}^{v}(t)$ is a scalar noise, $t=0,1, \ldots$, and $i=$ $1, \ldots K-1$. The extreme segments are described by

$$
\begin{aligned}
v_{0}(t+1) & =v_{0}(t)+\xi_{0}^{v}(t) \\
v_{K}(t+1) & =v_{K}(t)+\xi_{K}^{v}(t)
\end{aligned}
$$

where $t=0,1, \ldots ; \xi_{0}^{v}(t)$ and $\xi_{K}^{v}(t)$ scalar noises. Moreover, $\tau, \zeta, \nu$, and $\chi$ are positive constants and

$$
V\left[\rho_{i}(t)\right]=v_{\text {free }}\left\{1-\left[\frac{\rho_{i}(t)}{\rho_{\max }}\right]^{l}\right\}^{m}
$$

is the function representing the speed-density characteristic, with $l$ and $m$ positive real numbers. In addition, $v_{\text {free }}>0$ and $\rho_{\max }>0$ are the free speed and the critical density respectively.

Third, as to the percentage of active mobile phone, a technique of state augmentation is used, which leads to the definition of the following simple equation

$$
\delta_{i}(t+1)=\delta_{i}(t)+\xi_{i}^{\delta}(t)
$$




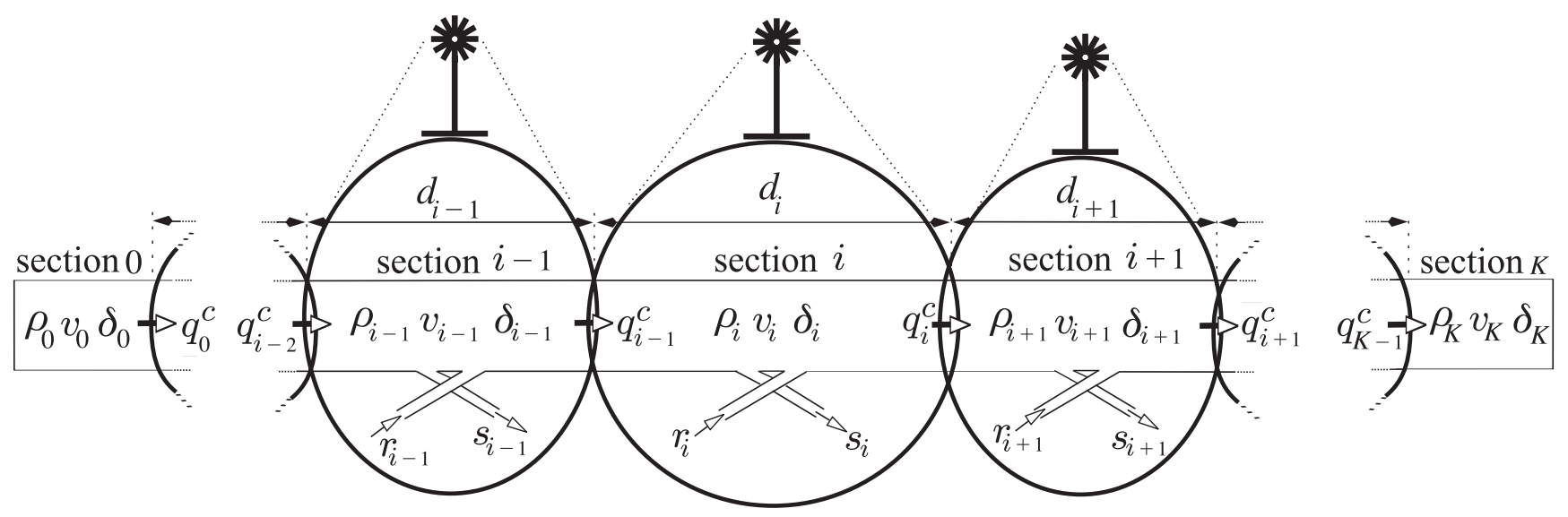

Fig. 1. A freeway trunk covered by cells of a mobile phone communication network.

where $t=0,1, \ldots$ and $i=0,1, \ldots, K$.

Note that equation (6a) is noise-free, as it results from the balance of the vehicles entering and leaving a section. On the contrary, (6b), (6c), (7a), (7b), (7c), and (8) are affected by disturbances. As to (7a), the noises represent uncertainties in the dynamics. In the other equations, such disturbances account for changes as, for example, in (8), where $\xi_{i}^{\delta}(t)$ models the change of percentage of active mobile phones.

We recall that measures of the variables $\rho_{i}^{c}(t)$ and $q_{i}^{c}(t)$ are available from the cellular network logging, as well as $r_{i}(t)$ and $s_{i}(t)$ can be easily computed in the on- and offramps. As a consequence, the measurement vector is given by

$$
\underline{y}(t)=\operatorname{col}\left[\rho_{1}^{c}(t), \ldots, \rho_{K-1}^{c}(t), q_{0}^{c}(t), \ldots, q_{K}^{c}(t)\right] \in \mathbb{R}^{2 K-1}
$$

where $t=0,1, \ldots$ and, for the real numbers $a$ and $b$, $\operatorname{col}(a, b) \triangleq(a b)^{\mathrm{T}}$. In addition, for the sake of compactness, let us define the state vector

$$
\begin{aligned}
\underline{x}(t)= & \operatorname{col}\left[\rho_{0}(t), \ldots, \rho_{K}(t), v_{0}(t), \ldots, v_{K}(t),\right. \\
& \left.\delta_{0}(t), \ldots, \delta_{K}(t)\right] \in \mathbb{R}^{3(K+1)} \\
\underline{\xi}(t)= & \operatorname{col}\left[\xi_{0}^{\rho}(t), \xi_{K}^{\rho}(t), \xi_{0}^{v}(t), \ldots, \xi_{K}^{v}(t),\right. \\
& \left.\xi_{0}^{\delta}(t), \ldots, \xi_{K}^{\delta}(t)\right] \in \mathbb{R}^{2 K+4} \\
\underline{q}_{c}(t)= & \operatorname{col}\left[q_{0}^{c}(t), \ldots, q_{K-1}^{c}(t)\right] \in \mathbb{R}^{K} \\
\underline{r}(t)= & \operatorname{col}\left[r_{i}(t), i \in I_{r}\right] \in \mathbb{R}^{\operatorname{card}\left(I_{r}\right)} \\
\underline{s}(t)= & \operatorname{col}\left[s_{i}(t), i \in I_{s}\right] \in \mathbb{R}^{\operatorname{card}\left(I_{s}\right)}
\end{aligned}
$$

Summing up, the overall dynamic model with state vector $\underline{x}(t)$ is given by equations (6), (7), and (8), which depend nonlinearly on the unknown parameters $\alpha, \tau, \nu, \chi, l, m$, $v_{\text {free}}$, and $\rho_{\max }$. For the sake of compactness, let us define

$$
\underline{p}=\operatorname{col}\left[\alpha, \tau, \nu, \chi, l, m, v_{\text {free }}, \rho_{\max }\right] .
$$

Let us denote, by means of the functions $f$ and $h$, the difference equation and algebraic mapping that represent the dynamics (via (6), (7), and (8)) and measurement equations (through (9)), i.e.,

$$
\begin{aligned}
\underline{x}(t+1) & =f\left[\underline{x}(t), \underline{r}(t), \underline{s}(t), \underline{q}_{c}(t), \underline{\xi}(t), \underline{p}\right] \\
\underline{y}(t) & =h[\underline{x}(t), \underline{p}]+\underline{\eta}(t)
\end{aligned}
$$

where $\underline{\eta}(t) \in \mathbb{R}^{2 K-1}$ is measurement noise vector.

In the next section, we shall address the problem of estimating the parameter vector $\underline{p}$.

\section{PARAMETER IDENTIFICATION}

System (10) provides a complete description of the proposed model, where the unknowns are the disturbances $\underline{\xi}(t)$ and $\underline{\eta}(t)$ as well as the parameter vector $\underline{p}$. According to a classical least-squares approach, $\underline{\xi}(t)$ and $\underline{\eta}(t)$ are supposed "small" and so one can try to estimate $\underline{p}$ by minimizing a regression cost function based on a batch of $N$ data collection, where the $n$-th is made of $T_{n}$ sample measurements $\underline{y}^{n}(t)$ and known inputs $\underline{r}^{n}(t), \underline{s}^{n}(t)$, and $\underline{q}_{c}^{n}(t)\left(t=1, \ldots, T_{n}\right)$. Moreover, we assume to know an estimate of the initial state $\underline{x}^{n}(0)$ given by $\underline{\bar{x}}^{n}(0)$.

Basing on the aforesaid, the predicted state trajectory is given by

$$
\underline{\hat{x}}^{n}(t+1)=f\left[\underline{\hat{x}}^{n}(t), \underline{r}^{n}(t), \underline{s}^{n}(t), \underline{q}_{c}^{n}(t), \underline{p}\right]
$$

where $n=1, \ldots N \quad t=0,1, \ldots, T_{n}-1, \underline{\hat{x}}^{n}(0)=\underline{\bar{x}}^{n}(0)$. A least-squares quadratic cost function that measures the quality of the fitting based on the available data is

$$
\begin{aligned}
J_{N}(\underline{p})= & \sum_{n=1}^{N} \sum_{t=1}^{T_{n}}\left\{\underline{y}^{n}(t)-h\left[\underline{\hat{x}}^{n}(t), \underline{p}\right]\right\}^{\mathrm{T}} R \times \\
& \times\left\{\underline{y}^{n}(t)-h\left[\underline{\hat{x}}^{n}(t), \underline{p}\right]\right\}
\end{aligned}
$$

where $R \in \mathbb{R}^{(2 K-1) \times(2 K-1)}$ is a positive definite matrix.

An estimate of $\underline{p}$ can be obtained by minimizing cost (12) under constraint (11), which turns out to be difficult due to the nonlinear dependence on $\underline{p}$. As the constraints can be taken into account by simply replacing (11) step by step from $t=0$ to $t=T_{n}-1$ for each $n=1, \ldots, N$, the problems reduces to the unconstrained minimization of $J_{N}(\underline{p})$, which can be faced by using the SPSA approach [10], [11], [12]. The use of such technique is motivated by the fact that the computation of the cost gradient is in general difficult in our context.

Let $p$ be the dimension of $\underline{p}$. The SPSA algorithm has the form

$$
\underline{p}_{k+1}=\underline{p}_{k}-a_{k} \hat{g}_{k}\left(\underline{p}_{k}\right), k=0,1, \ldots
$$


where $\hat{g}_{k}\left(\underline{p}_{k}\right)$ is a is a simultaneous perturbation approximation of the gradient of the cost at iteration $k$, i.e., of $\nabla J_{N}\left(\underline{p}_{k}\right)$. The simultaneous perturbation approximation is defined as follows. Let $\Delta_{k} \in \mathbb{R}^{p}$ be a vector of $p$ mutually independent mean zero random variables $\left\{\Delta_{k_{1}}, \Delta_{k_{2}}, \ldots, \Delta_{k_{p}}\right\}$. In the general framework of stochastic approximations, we refer to noisy measurements of the cost function, i.e., at the $k$-th iteration, we have

$$
\begin{aligned}
J_{k}^{+}\left(\underline{p}_{k}\right) & =J_{N}\left(\underline{p}_{k}+c_{k} \Delta_{k}\right)+\epsilon_{k}^{+} \\
J_{k}^{-}\left(\underline{p}_{k}\right) & =J_{N}\left(\underline{p}_{k}-c_{k} \Delta_{k}\right)+\epsilon_{k}^{-}
\end{aligned}
$$

where $\left\{c_{k}\right\}$ is a gain sequence, while $\epsilon_{k}^{+}$and $\epsilon_{k}^{-}$are measurement noises. The estimate of $\nabla J_{N}\left(\underline{p}_{k}\right)$ is given by

$$
\hat{g}_{k}\left(\underline{p}_{k}\right)=\left[\begin{array}{c}
\frac{J_{k}^{+}\left(\underline{p}_{k}\right)-J_{k}^{-}\left(\underline{p}_{k}\right)}{2 c_{k} \Delta_{k_{1}}} \\
\vdots \\
\frac{J_{k}^{+}\left(\underline{p}_{k}\right)-J_{k}^{-}\left(\underline{p}_{k}\right)}{2 c_{k} \Delta_{k_{p}}}
\end{array}\right]
$$

If we assume the following, where $E(\cdot)$ stands for the expected value with respect to all the stochastic variables.

A1. $a_{k}=\frac{a}{k^{\alpha}}$ and $c_{k}=\frac{1}{k^{\gamma}}$, where $a>0,0<\alpha \leq 1$, $\gamma>0, \alpha-\gamma>\frac{1}{2}, \alpha-2 \gamma>0,3 \gamma-\frac{\alpha}{2} \geq 0$.

A2. for all $k=0,1, \ldots, E\left(\epsilon_{k}^{+}-\epsilon_{k}^{-} \mid \underline{p}_{k}, \Delta_{k}\right)=$ 0 ; there exists $\nu>0$ such that $E\left[\left(\epsilon_{k}^{-}\right)^{2+\nu}\right]$ $E\left[\left(\epsilon_{k}^{+}\right)^{2+\nu}\right]$ are bounded; there exists $\sigma$ such that $\lim _{k \rightarrow+\infty} E\left[\left(\epsilon_{k}^{+}-\epsilon_{k}^{-}\right)^{2} \mid \underline{p}_{k}, \Delta_{k}\right]=\sigma^{2}$.

A3. for all $k=0,1, \ldots,\left\{\Delta_{k_{i}}\right\}(i=1, \ldots, p)$ are i.i.d. and symmetrically distributed around zero with $\left\|\Delta_{k_{i}}\right\|$ bounded a.s. and $E\left(\left|\Delta_{k_{i}}^{-1}\right|\right)$ bounded; there exists $\nu>0$ such that $E\left(\left|J_{N}\left(\underline{p}_{k}-c_{k} \Delta_{k}\right)\right|^{2+\nu}\right)$, $E\left(\left|J_{N}\left(\underline{p}_{k}+c_{k} \Delta_{k}\right)\right|^{2+\nu}\right)$, and $E\left(\left|\Delta_{k_{i}}\right|^{-2-\nu}\right)(i=$ $1, \ldots, p)$ are bounded; there exist $\sigma_{-}$and $\sigma_{+}$such that $\lim _{k \rightarrow+\infty} E\left(\Delta_{k_{i}}^{-2}\right)=\sigma_{-}^{2}$ and $\lim _{k \rightarrow+\infty} E\left(\Delta_{k_{i}}^{2}\right)=\sigma_{+}^{2}$, $i=1, \ldots, p$.

A4. The third derivative of $J_{N}(\underline{p})$ with respect to $\underline{p}$ is locally continuous and bounded for almost all $\underline{p}_{k}, \bar{k}=$ $0,1, \ldots$ in a ball with radius independent of $k$ and $\underline{p}_{k}$.

Basing on the above assumptions, it is possible to prove that $k^{\beta / 2}\left(\underline{p}_{k}-\underline{p}^{*}\right)$ converges in distribution a Gaussian distributed variable, where $\underline{p}^{*}$ is a root of $\frac{\partial J_{N}(\underline{p})}{\partial p}$ and $\beta>0$ is constant (see, for details, [15] and the references therein).

\section{Simulation RESUlts}

In order to evaluate the performance of the proposed approach, a complex simulation environment has been devised with a microscopic traffic simulator and a simulator of the cellular network. Fig. 2 shows a sketch of such simulation setup.
The behavior of multiple cars moving in a freeway is emulated by a microscopic simulator based on the Gipps' model [16]. This simulator generates the positions of the cars that can open wireless communications.

The simulator of the cellular system is protocol independent since it is based on a Markov model of the events associated with the events regarding start of a call, end of a call, and handoff from one cell to the next one. Such model does not rely on a specific architecture (e.g., ETACS, GSM, UMTS), as well as either circuit or packet switching may be considered. The wireless network manages the radio-based links, tracks the , and provides information on both density of active mobile phone in each cell and flow from one cell to the next one. A linear relationship between the level of freeway traffic and the network load is assumed (see [17]).

The handoff of a call occurs when the associated vehicle passes from one cell to another. As in most cellular systems, users are registered when they enter a cell, the management system keeps track of the number of all mobiles present inside (i.e., $\rho_{i}^{c}(t)$ for $i$-th cell). In the same way, it is possible to count the mobile phones leaving a cell (i.e., $q_{i}^{c}(t)$ for $i$-th cell), which corresponds to the exiting handoffs of the calls.

A simulation setting was considered with a 3-lane freeway trunk, where we chose $K=11,2 \mathrm{Km}$-long sections, $\Delta T=$ $60 \mathrm{~s}$, and all $T_{n}$ equal to 120 (i.e., $2 \mathrm{~h}$ ). The percentage of active mobile phone is randomly chosen in each simulation according to a uniform distribution with percentage between $10 \%$ and $30 \%$.

The SPSA identification method presented in Section III has been applied to a cost function (12), where $R$ has been chosen equal to the identity and the algorithm parameters were taken as in Assumptions A1, A2, A3, and A4.

Fig. 3 shows the behavior of the cost function $J_{N}$ at each iteration $k$ for different values of the number of realization $N$ in the the sample set. The larger is $N$, the higher is the computational burden and the more significative is the sample set. In Fig. 4, the estimates of the parameters are given at each iteration for $N=10,50$, and 100 . Note that the convergence of both cost function and estimated parameters is faster with a larger $N$. Some parameters as $\tau$ and $m$ are very slow to converge with $N=10$.

\section{Conclusions}

In this paper, a modelling framework is presented that enables one to describe the traffic dynamics using the measurements collected by the active mobile phones on the freeway cars. This model results from an extension of the dynamic macroscopic traffic model D (see [1]) that includes other dynamic equations about the percentage of active mobile phones in the various segments of a freeway trunk. Using such framework, one can estimate the model parameters via an identification algorithm based on stochastic approximation. The overall approach has been validated by constructing a complex simulation setup, with encouraging results.

In the future, the identification of such model will be used in connection with state estimators that allow a complete 


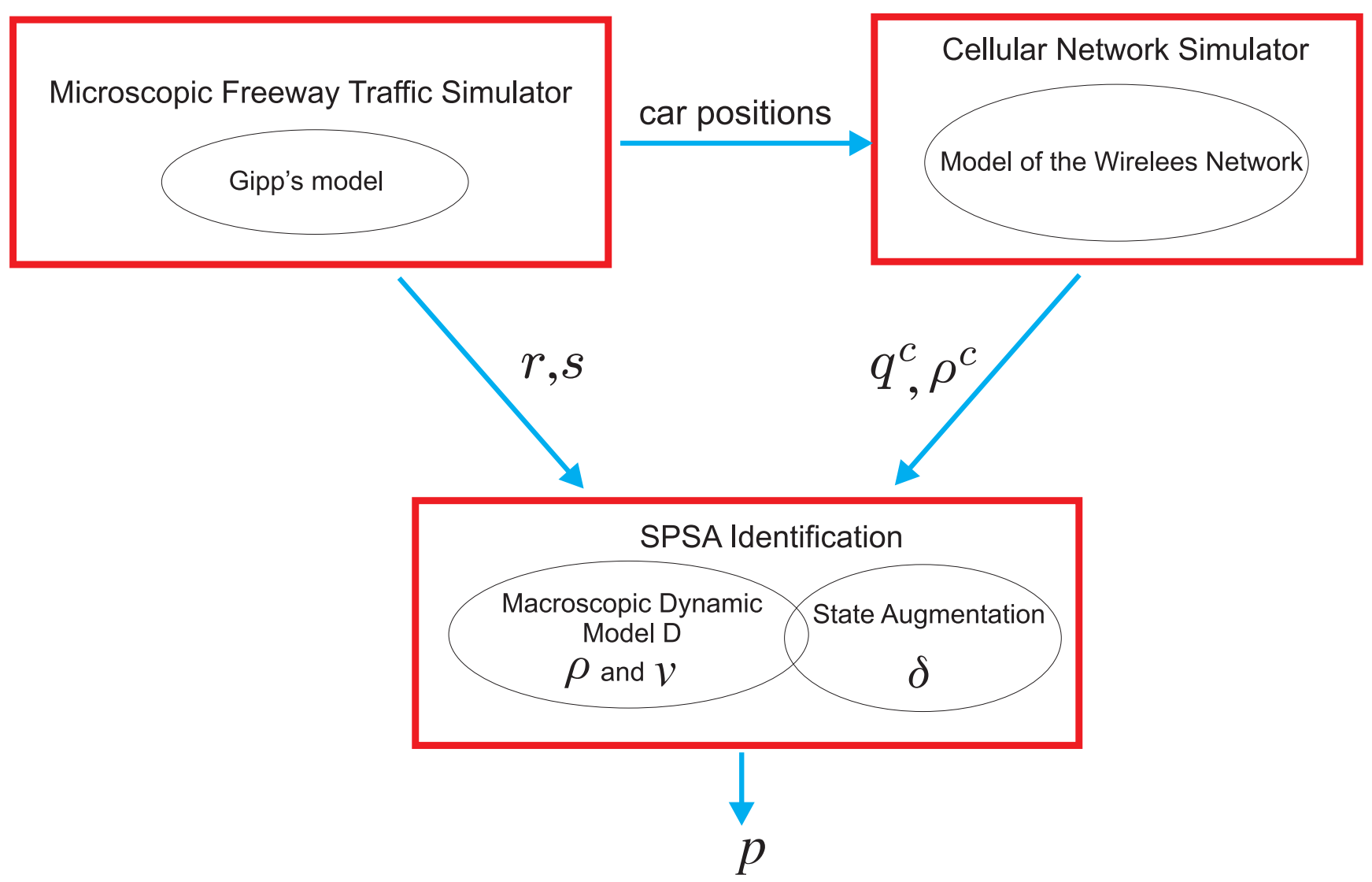

Fig. 2. Simulation setup.

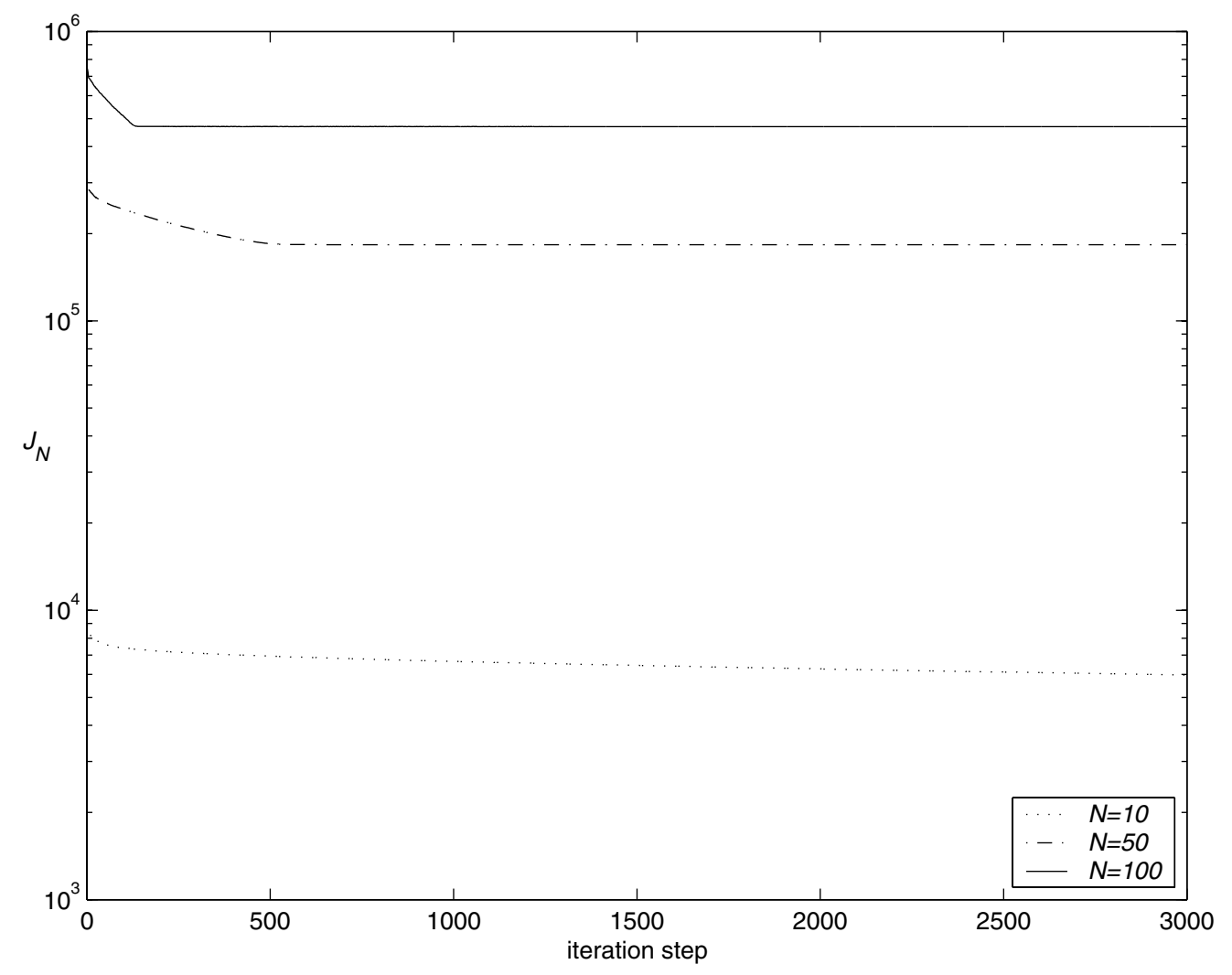

Fig. 3. Cost functions 

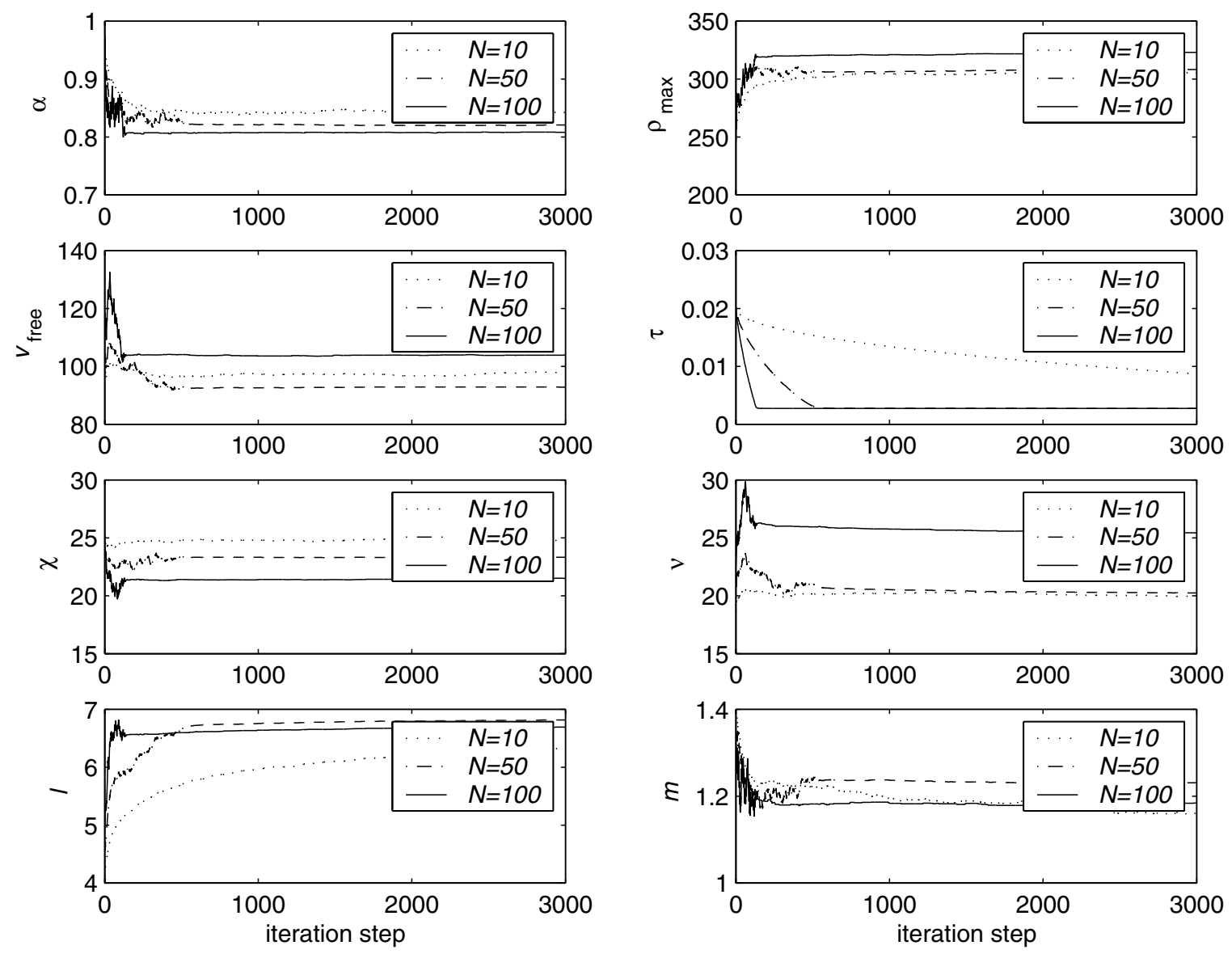

Fig. 4. Parameter estimates

supervision of the traffic state (see, e.g., [18], [19], [20]).

\section{REFERENCES}

[1] M. Papageorgiou, "Applications of Automatic Control Concepts to Traffic Flow Modeling Control," in Lecture Notes in Control and Information Sciences, A. Balakrishnan and M. Thoma, Eds. Berlin: Springer-Verlag, 1983, vol. 50.

[2] L. Isaksen and H. J. Payne, "Freeway traffic surveillance and control," Proceedings of the IEEE, vol. 61, pp. 526-536, 1973.

[3] H. J. Payne, W. A. Thompson, and L. Isaksen, "Design of a trafficresponsive control system for a los angeles freeway," IEEE Trans. on Systems, Man, and Cybernetics, vol. 3, pp. 213-224, 1973.

[4] M. Y. Blinkin, "Problems of optimal control of traffic flow on highways," Automation and Remote Control, vol. 37, pp. 662-667, 1976.

[5] D. P. Looze, P. K. Houpt, N. R. Sandell, and M. Athans, "On decentralized estimation and control with application to freeway ramp metering," IEEE Trans. on Automatic Control, vol. 23, pp. 268-275, 1978.

[6] S. A. Smulders, "Control of freeway traffic flow by variable speed signs," Transpn. Res. B, vol. 24B, no. 2, pp. 111-132, 1990.

[7] M. Papageorgiou, "Freeway ramp metering: an overview," IEEE Trans. on Intelligent Transportation Systems, vol. 3, no. 4, pp. 271-281, 2002.

[8] M. Cremer and M. Papageorgiou, "Parameter identification for a traffic flow model," Automatica, vol. 17, no. 7, pp. 837-843, 1981.

[9] K. K. Sanwal, K. Petty, J. Walrand, and Y. Fawaz, "An extended macroscopic model for traffic flow," Transportation Research B, vol. 30, no. 1, pp. 1-99, 1996.

[10] J. Spall, "Multivariate stochastic approximation using a simultaneous perturbation gradient approximation," IEEE Trans. on Automatic Control, vol. 37, no. 3, pp. 332-341, 1992.
[11] L. Gerencser, "Convergence rate of moments in stochastic approximation with simultaneous perturbation gradient approximation and resetting," IEEE Trans. on Automatic Control, vol. 44, no. 5, pp. 894905, 1999.

[12] J. Spall, "Adaptive stochastic approximation by the simultaneous perturbation method," IEEE Trans. on Automatic Control, vol. 45, no. 10 , pp. 1839-1853, 2000.

[13] C. Setchell and E. L. Dagless, "Vision-based road-traffic monitoring sensor," IEE Proc.-Vis. Image Signal Process., vol. 148, no. 1, pp. 78-84, 2001.

[14] A. Alessandri and T. Parisini, "Nonlinear modeling of complex largescale plants using neural networks and stochastic approximation," IEEE Trans. on Systems, Man, and Cybernetics-Part A: Systems and Humans, vol. 27, no. 6, pp. 750-757, 1997.

[15] P. Sagdegh and J. Spall, "Optimal random perturbations for stochastic approximation using a simultaneous perturbation gradient approximation," IEEE Trans. on Automatic Control, vol. 43, no. 10, pp. 14801484, 1998.

[16] P. G. Gipps, "A behavioural car-following model for computer simulation," Transportation Research B, vol. 15, pp. 105-111, 1981.

[17] G. Montenegro, M. Sengoku, Y. Ymaguchi, and T. Abe, "Timedependent analysis of mobile communication traffic in a ring-shaped service area with nonuniform vehicle density," IEEE Trans. on Vehicular Technology, vol. 41, no. 3, pp. 243-254, 1992.

[18] M. Wirth and U. Brunner, "Observer models for real-time traffic control," in American Control Conference, Baltimore, Maryland, June 1994.

[19] A. Alessandri, R. Bolla, and M. Repetto, "Estimation of freeway traffic variables using information from mobile phones," in American Control Conference, Denver, CO, 2003, pp. 4089-4094.

[20] Y. Wang and M. Papageorgiou, "Real-time freeway traffic state estimation based on the extended Kalman filter: a general approach," Transportation Research, Part B, vol. 39, pp. 141-167, 2005. 